\title{
Disentangling rotational velocity distribution of stars
}

\author{
Michel Curé ${ }^{1}$, Diego F. Rial ${ }^{2}$, Julia Cassetti ${ }^{3}$ and Alejandra Christen ${ }^{4}$ \\ ${ }^{1}$ Universidad de Valparaíso, Chile \\ ${ }^{2}$ Universidad de Buenos Aires, Argentina \\ ${ }^{3}$ Universidad Nacional de General Sarmiento, Argentina \\ ${ }^{4}$ Pontificia Universidad Católica de Valparaíso, Chile
}

\begin{abstract}
Rotational speed is an important physical parameter of stars: knowing the distribution of stellar rotational velocities is essential for understanding stellar evolution. However, rotational speed cannot be measured directly and is instead the convolution between the rotational speed and the sine of the inclination angle $v \sin (i)$. The problem itself can be described via a Fredhoml integral of the first kind. A new method (Curé et al. 2014) to deconvolve this inverse problem and obtain the cumulative distribution function for stellar rotational velocities is based on the work of Chandrasekhar \& Münch (1950). Another method to obtain the probability distribution function is Tikhonov regularization method (Christen et al. 2016). The proposed methods can be also applied to the mass ratio distribution of extrasolar planets and brown dwarfs (in binary systems, Curé et al. 2015).

For stars in a cluster, where all members are gravitationally bounded, the standard assumption that rotational axes are uniform distributed over the sphere is questionable. On the basis of the proposed techniques a simple approach to model this anisotropy of rotational axes has been developed with the possibility to "disentangling" simultaneously both the rotational speed distribution and the orientation of rotational axes.
\end{abstract}

Keywords. methods: analytical - methods: data analysis - methods: numerical - methods: statistical - stars: fundamental- parameters - stars: rotation

\section{Results}

We have assumend tha the rotational velocity distribution is given by a Maxwellian distribution and integrate analytically the Fredhoml integral. The rotational data have been selected for twelve clusters from the survey of radial and rotational velocities performed by Mermilliod et al. (2009). We fitted (1000 bootstraps) to the distribution of projected velocity with our formula (from Fedhom integral) for a single Maxwellian distribution, but also for a double Maxwellian distribution.

In all cases we find that the distribution of rotational axes is not randomly oriented. The results also show that a mixture of two Maxwellian distribution fits better the observed projected velocity sample. This might indicate the existence of two stellar populations.

\section{References}

Chandrasekhar, S. \& Münch, G. 1950, ApJ, 111, 142

Christen, A, Escarate, P., Curé, M., Rial, D. F., \& Cassetti, J. 2016, A\&A , 595, 50

Curé, M., Rial, D. F., Christen, A., \& Cassetti, J. 2014, A\&A , 565, 85

Curé, M., Rial, D. F., Cassetti, J., Christen, A., \& Boffin, H. M. J. 2015, A\&\&A, 573, 86

Mermilliod, J.-C., Mayor, M., \& Udry, S., 2009, A\&A, 498, 949 